\title{
Impact of Home Oxygen Therapy on the Level of Physical Activities in Daily Life in Subjects With COPD
}

\author{
Katerine C Cani, Darlan L Matte, Isabela J C S Silva, Aline A Gulart, Manuela Karloh, and \\ Anamaria F Mayer
}

\begin{abstract}
BACKGROUND: Chronic hypoxemia in patients with COPD is a limiting factor that is associated with disease progression and significantly contributes to decreased physical activity. Although correction of hypoxemia results in physiological improvements, oxygen therapy may be related to social isolation and inactivity. However, few studies have objectively investigated the level of physical activity in daily life (PADL) in these patients. The objective of this study was to compare the level of PADL in subjects with COPD on home oxygen therapy and in subjects with no oxygen therapy, and to investigate whether home oxygen therapy is associated with the level of PADL. METHODS: In this study, 29 subjects with COPD composed the home oxygen therapy group, and 30 subjects with COPD composed the control group. All of them underwent assessment of anthropometric measurements, spirometry, peripheral muscle strength, limitations in activities of dalily living, perception of dyspnea, health status, as well as PADL monitoring. RESULTS: Home oxygen therapy subjects showed less time walking $(P=.001)$, in active physical activities $(P=.003)$, in physical activities $\geq 3$ METs $(P=.001)$, and in physical activities $<1.5$ METs $(P=.042)$, as well as fewer steps $(P=.001)$. Physical inactivity was present in 24 home oxygen subjects $(82.8 \%)$ and 18 control subjects $(60 \%)$. Oxygen therapy was associated with severe physical inactivity (Cramer's $\mathrm{V}=0.29, P=.040)$. The time using oxygen therapy $(\mathrm{h} / \mathrm{d})$ was the strongest predictor of the level of PADL $\left(\mathrm{r}^{2}=0.38-0.43, P<.001\right)$. CONCLUSION: Subjects with COPD using oxygen at home showed reduced level of PADL. The results indicate that oxygen therapy is associated with severe physical inactivity and may be a predictor of the level of PADL. Key words: COPD; motor activity; oxygen inhalation therapy; home visit; pulmonary disease; mobility limitation. [Respir Care 2019;64(11):1392-1400. () 2019 Daedalus Enterprises]
\end{abstract}

\section{Introduction}

In more advanced stages of COPD, there is a notable decrease in physical activity in daily life (PADL), and patients are limited by specific disease factors such as

\footnotetext{
All of the authors are affiliated with the Núcleo de Assistência, Ensino e Pesquisa em Reabilitação Pulmonar, Universidade do Estado de Santa Catarina, Florianópolis, Santa Catarina, Brazil. Ms Cani, Mr Matte, Ms Karloh, and Dr Mayer are affiliated with the Programa de Pós-Graduação em Fisioterapia, Centro de Ciências da Saúde e do Esporte, Universidade do Estado de Santa Catarina, Florianópolis, Santa Catarina, Brazil. Ms Silva is affiliated with the Curso de graduação em Fisioterapia da Universidade do Estado de Santa Catarina, Florianópolis, Santa Catarina, Brazil. Ms Gulart and Dr Mayer are affiliated with the Programa de Pós-Graduação em Ciências do Movimento Humano, Centro de Ciências da Saúde e do Esporte, Universidade do Estado de Santa Catarina, Florianópolis, Santa Catarina, Brazil.
}

pulmonary hyperinflation, severe dyspnea, worse lowerlimb muscle function, and chronic hypoxemia. ${ }^{1}$ At these stages of COPD, home oxygen therapy is usually part of the nonpharmacological treatment for hypoxemia. ${ }^{2}$ This therapy may improve survival, quality of life, pulmonary

\footnotetext{
Ms Cani presented a version of this paper as an abstract at the XVIII Simpósio Internacional de Fisioterapia Cardiorrespiratória e Fisioterapia em Terapia Intensiva, held June 8-11, 2016, in Belo Horizonte, Brazil.

The authors have disclosed no conflicts of interest.

Correspondence: Anamaria F Mayer PhD, Physiotherapy Department, Universidade do Estado de Santa Catarina, Rua Pascoal Simone 358, 88080-350, Florianópolis, Brazil. E-mail: anamaria.mayer@udesc.br.
}

DOI: $10.4187 /$ respcare.06206 


\section{Home Oxygen and Physical Activity in COPD}

hemodynamics, exercise capacity, and cognitive performance in these patients, ${ }^{3,4}$ and it may reduce pulmonary hypertension and, subsequently, the risk of cor pulmonale. ${ }^{5}$

Oxygen supplementation results in improved exercise tolerance in patients who are severely incapacitated by advanced COPD because it slows the development of ventilatory limitation, thus promoting a reduction in ventilatory demand and, consequently, dynamic hyperinflation and dyspnea on exertion. In addition to reducing the overload of the respiratory system, oxygen supplementation improves the supply of oxygen to the muscles, thus slowing the onset of fatigue. ${ }^{2,6,7}$

Despite its positive effects, studies have reported that the use of oxygen therapy may be associated with low levels of $\mathrm{PADL}^{8,9}$ and that subjects using home oxygen therapy showed less independence activities of daily living. ${ }^{10}$ Some investigators have also reported an association among higher frequency of exacerbations, lower functional capacity, lower motivation to exercise, and reduced quality of life during the time such subjects remain seated in daily life, with a higher proportion in subjects using home oxygen therapy. ${ }^{1}$ They also present a higher number of hospital admissions, symptoms of anxiety and depression, and worse mortality prognosis. ${ }^{11}$

Although the use of oxygen therapy has been previously associated with inactivity, only one study ${ }^{12}$ used triaxial accelerometers, which are more reliable in assessing the level of PADL. ${ }^{13-15}$ However, that study included a small sample of home oxygen users and considered only a few variables related to PADL. Other studies were performed using scales and uniaxial accelerometers, which are less reliable methods to evaluate PADL. ${ }^{8-10}$ Moreover, no study has so far compared the level of PADL among subjects with COPD on oxygen therapy or not on oxygen therapy, matching them according to pulmonary function.

This study aimed to compare the level of PADL in subjects with COPD on home oxygen therapy and those not on oxygen therapy (matched according to pulmonary function), and to investigate whether the use of home oxygen therapy is associated with the level of PADL.

\section{Methods}

\section{Subjects}

In this study, the participants were classified into a group of subjects with COPD using home oxygen therapy or a group of controls with COPD not using oxygen therapy. They were matched according to sex, age, body mass index, and percent of predicted $\mathrm{FEV}_{1}$ recruited from the Home Oxygen Therapy Services of the Municipal Health Department of Florianópolis and São José, and from public and private out-patient clinics in the metropolitan area

\section{QUICK LOOK}

\section{Current knowledge}

The use of oxygen therapy may be associated with low levels of physical activity in patients with COPD. The level of physical activity is also strong predictor of mortality in patients with COPD. The use of home oxygen therapy is also associated with lower functional capacity, lower motivation to exercise, reduced quality of life during the time they remain seated in daily life, and worse prognosis.

\section{What this paper contributes to our knowledge}

Subjects with COPD on home oxygen therapy demonstrated lower levels of physical activity in daily life (PADL), greater limitations and higher levels of dependence in PADL, greater perception of dyspnea, and worse general health status compared to subjects who do not use oxygen therapy and have similar lung function. The duration of oxygen therapy in terms of hours/day was the strongest predictor of PADL in this population.

of Florianópolis, SC/Brazil. This study was approved by the Human Research Ethics Committee of the State University of Santa Catarina (CAAE: 42993115.7.0000. 0118). All participants signed an informed consent document.

Inclusion criteria for the home oxygen therapy group were clinical diagnosis of COPD in clinically stable GOLD spirometric stages III and IV in the month prior to the start of the protocol; ${ }^{16}$ use of home oxygen therapy at a minimum during sleep, and for at least 3 months; ${ }^{17}$ age between 40 and $80 \mathrm{y}$; and a history of smoking at least 10 packs per year with smoking cessation for more than 6 months. Exclusion criteria were inability to execute the proposed protocol; comorbidities that influenced or could limit PADL (cardiovascular, neurological, orthopedic, and/or rheumatologic comorbidities); other chronic respiratory diseases; participation in a pulmonary rehabilitation program for $<6$ months; and exacerbation of the disease during the period of data collection. The same criteria were adopted for the control group, with the exception of the use of home oxygen therapy.

\section{Sample Size Calculation}

The sample size was calculated using the mean walking time found in the pilot study of 10 home oxygen therapy group subjects and 10 control group subjects $(44.8 \pm 24.6 \mathrm{~min}$ vs $89.2 \pm 40.3 \mathrm{~min}$, respectively) 


\section{Home Oxygen and Physical Activity in COPD}

(G*Power 3.1.9.2). Considering a $t$ test, a bidirectional alpha of 0.05 and beta of 0.20 , and sample loss of 20\%, a sample size of 16 subjects per group was obtained. We estimated a total of 29 subjects for each group, expecting correlation coefficients of at least 0.5 with the same values of alpha, beta, and loss. ${ }^{18}$

\section{Protocol}

This was an observational cross-sectional study carried out in $3 \mathrm{~d}$ of evaluation. On the first day, anthropometry measurements, pulmonary function, oxygen therapy characteristics, functional status, peripheral muscle strength, perception of dyspnea, and health status were evaluated. On the second and third days, PADL was monitored. Oxygen therapy was maintained according to the habitual use throughout the protocol, observing the schedules and flows already established.

Characteristics related to the use of home oxygen therapy included period of use (eg, nocturnal or nocturnal/diurnal); oxygen flow ( $\mathrm{L} / \mathrm{min}$ ); time of oxygen therapy (in $\mathrm{h} / \mathrm{d}$ and $\mathrm{y}$ ); oxygen saturation $\left(\mathrm{S}_{\mathrm{pO}_{2}}\right)$ at rest; use of portable devices or lack of such use; tubing lengths, if the subject received medical advice to alter the flow of oxygen at any time of the day or for any specific reason (such as during a physical activity or in exacerbations of the disease); and if the subject followed medical prescriptions (eg, time of use and oxygen flow). Long-term administration of oxygen was defined as the use for $>15 \mathrm{~h}^{2}$ Thus, the time of oxygen use was classified as nocturnal, nocturnal/diurnal $\leq 15 \mathrm{~h}$, and nocturnal/diurnal $>15 \mathrm{~h}$.

Pulmonary function was assessed with spirometry using the EasyOne spirometer (ndd Medical Technologies, Switzerland), and the calibration was checked at each assessment. We adopted methods and criteria recommended by the American Thoracic Society/European Respiratory Society ${ }^{19}$ and the reference values of Pereira et al. ${ }^{20}$ Dyspnea on exertion was assessed with the modified Medical Research Council scale, ${ }^{21,22}$ and health status was assessed with the COPD Assessment Test. ${ }^{23}$

Peripheral muscle strength of knee extensors was evaluated as described by Andrews et $\mathrm{al}^{24}$ using a portable digital dynamometer (Lafayette Instrument, Lafayette, Indiana). At least 5 and at most 8 repetition were performed, 2 of which should be reproducible (difference of $\leq 10 \%$ ). ${ }^{24}$ The analyzed variable was the mean of the isometric strength in Newtons $(\mathrm{N})$ of the two best reproducible measures.

Functional limitations were evaluated with the modified version of the Pulmonary Functional Status and Dyspnea Questionnaire, which is composed of three domains: dyspnea influence on PADL, fatigue influence on PADL, and change in PADL compared to period prior to disease. The total score was used for the analyses. ${ }^{20}$ To assess the level of dependence for PADL, the Katz index was applied and the total score was considered. ${ }^{25,26}$

PADL were monitored with a triaxial accelerometer (DynaPort Activity Monitor, McRoberts, The Netherlands) for $12 \mathrm{~h}$ on 2 consecutive days after waking up. ${ }^{27,28}$ The time spent standing, sitting, lying down, and walking, as well as the intensity of movement during walking ${ }^{29}$ and energy expenditure in PADL ${ }^{30}$ were used for the analyses. The sum of the times walking and standing composes the time variable in active physical activities, whereas the sum of the time lying down and sitting makes up the time in sedentary activities. Subjects with $<4,580$ steps were classified as severe physical inactivity. ${ }^{31}$ The time spent on physical activities with metabolic equivalent of task (MET) $\geq 3$ METs was measured, and the cut-off point of $80 \mathrm{~min} / \mathrm{d}$ was used to classify the subjects according to their level of physical (in)activity. The time spent on sedentary behaviors was also assessed considering physical activities $<1.5$ METs. ${ }^{32}$ Subjects were classified as sedentary and nonsedentary according to the cutoff point of $8.5 \mathrm{~h}^{33}$

\section{Statistical Analysis}

The data were analyzed with the IBM SPSS Statistics 20.0. The values are expressed as arithmetic mean, SD, median, 95\% CI, and percentile (interquartile range) according to the type of variables and distribution of data, details that were verified using the Shapiro-Wilk test. The chi-square test was used to compare the frequency of the GOLD stages between the groups and to test the association between the use of oxygen therapy and the level of PADL. The Cramer's V coefficient demonstrated the strength of these associations. For the comparisons between the groups (home oxygen therapy group and control group) and between the GOLD stages (III and IV), the Student $t$ test for independent samples or the Mann-Whitney $U$ test were used. Pearson or Spearman correlation coefficients were used to verify the correlation between the time of use of oxygen therapy (h/d), lung function and the PADL variables. Single linear and multiple stepwise linear regressions were applied using the variables related to PADL as dependent, and the variables related to oxygen therapy as independent. The significance level was set at $P<.05$.

\section{Results}

A total of 162 patients were recruited for the home oxygen therapy group, 30 of whom were eligible. One subject was excluded for not being able to complete the pulmonary function test. Thus, 29 subjects ( $21 \mathrm{men})$ completed the protocol in the home oxygen therapy group. For the control group, from 66 patients recruited, 30 subjects were eligible and finalized the study. Subjects on home 
Table 1. Sample Characterization

\begin{tabular}{|c|c|c|c|}
\hline & $\begin{array}{c}\text { Domiciliary } \\
\text { Oxygen } \\
\text { Therapy } \\
\text { Group }\end{array}$ & $\begin{array}{l}\text { Control } \\
\text { Group }\end{array}$ & $P$ \\
\hline Age, y & $68.3 \pm 7.72$ & $67.50 \pm 7.61$ & .67 \\
\hline Weight, kg & $66.2 \pm 16.3$ & $63.7 \pm 13.5$ & .52 \\
\hline Height, m & $1.63 \pm 0.10$ & $1.65 \pm 0.09$ & .30 \\
\hline Body mass index, $\mathrm{kg} / \mathrm{m}^{2}$ & $24.7 \pm 4.48$ & $23.2 \pm 4.31$ & .18 \\
\hline Smoking history, pack-years & $57.3 \pm 30.3$ & $61.7 \pm 36.1$ & .78 \\
\hline $\begin{array}{l}\text { Exacerbation in the } \\
\text { previous year }\end{array}$ & $2.28 \pm 1.89$ & $1.19 \pm 1.54$ & .01 \\
\hline $\mathrm{FEV}_{1} / \mathrm{FVC}, \mathrm{L}$ & $0.37 \pm 0.09$ & $0.37 \pm 0.07$ & .60 \\
\hline $\mathrm{FEV}_{1}, \mathrm{~L}$ & $0.70 \pm 0.25$ & $0.73 \pm 0.29$ & .94 \\
\hline $\mathrm{FEV}_{1}, \%$ pred. & $24.6 \pm 6.71$ & $24.4 \pm 8.55$ & .90 \\
\hline FVC, L & $1.91 \pm 0.65$ & $1.92 \pm 0.56$ & .95 \\
\hline FVC, \%pred. & $52.1 \pm 12.3$ & $50.3 \pm 11.8$ & .50 \\
\hline GOLD III, $n(\%)$ & $6(20.7)$ & $6(20.0)^{*}$ & .60 \\
\hline GOLD IV, $n(\%)$ & $23(79.3)$ & $24(80.0)^{*}$ & \\
\hline Peripheral muscle strength, $\mathrm{N} \dagger$ & $142 \pm 41.0$ & $159 \pm 41.2$ & .19 \\
\hline PSFDQ total $\dagger$ & $100.6 \pm 69.8$ & $56.1 \pm 48.4$ & .030 \\
\hline $\mathrm{mMRC} \ddagger$ & $4(3-4)$ & $2(1-3)$ & $<.001$ \\
\hline Katz Index $\dagger+$ & $0(0-2)$ & $0(0-0)$ & .040 \\
\hline COPD Assessment Test & $23.3 \pm 8.48$ & $17.6 \pm 8.36$ & .01 \\
\hline Resting $\mathrm{S}_{\mathrm{pO}_{2}}$ & $95.4 \pm 3.70$ & $94.5 \pm 2.60$ & .65 \\
\hline \multicolumn{4}{|c|}{ 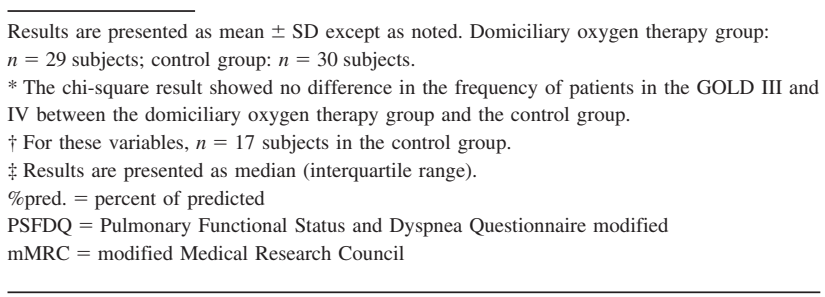 } \\
\hline
\end{tabular}

oxygen presented higher dyspnea, worse health status, greater number of exacerbations in the past year, and worse functional status related to PADL $(P<.05)$. The characteristics of the subjects are summarized in Table 1 , and the characteristics of the home oxygen therapy in Table 2.

Subjects on home oxygen therapy reported less time spent on active physical activities $(P=.003)$ and physical activities $\geq 3$ METs compared to control group $(P=.001)$ (Table 3 and Fig. 1). PADL differed between GOLD III and GOLD IV subjects only in the control group (Table 3). In the home oxygen therapy group, 4 subjects $(14.3 \%)$ were classified as active, 24 subjects $(82.8 \%)$ showed severe physical inactivity, and only 1 subject (3.6\%) did not exhibit sedentary behaviors. In the control group, 10 subjects $(33.3 \%)$ were classified as active, 18 subjects $(60 \%)$ showed severe physical inactivity, and 2 subjects $(6.7 \%)$ presented with no sedentary behaviors. The use of oxygen therapy was associated with the classification of severe physical inactivity (Cramer's V $=0.29, P=.040$ ).

As observed in Table 4, the time of oxygen therapy (h/d) was able to explain $41 \%$ of the variability in walking
Table 2. Characteristics Related to Oxygen Therapy in the Domiciliary Oxygen Therapy Group $(n=29)$

\begin{tabular}{lc}
\hline \hline & $n(\%)$ \\
\hline Oxygen therapy & \\
$\quad$ Night & $5(17.2)$ \\
Night and daytime $(\geq 15 \mathrm{~h})$ & $20(69.0)$ \\
$\quad$ Night and daytime $(<15 \mathrm{~h})$ & $4(13.8)$ \\
$\mathrm{O}_{2}$ flow, $\mathrm{L} / \mathrm{min}^{*}$ & $2(2-2.5)$ \\
Duration of oxygen therapy, $\mathrm{h} / \mathrm{d} *$ & $20(11-24)$ \\
Duration of oxygen therapy, $\mathrm{y}^{*}$ & $4(0.4-5.3)$ \\
Tubing length, m* & $9.5(2.1-9.6)$ \\
Orientation to change $\mathrm{O}_{2}$ flow & \\
$\quad$ Yes & $8(27.6)$ \\
$\quad$ No & $21(72.4)$ \\
Portable fountain & $11(37.9)$ \\
$\quad$ Yes & $18(62.1)$ \\
$\quad$ No & \\
Follows medical prescription & $25(86.2)$ \\
$\quad$ Yes & $4(13.8)$ \\
No & \\
\hline Results are presented as median (interquartile range). & \\
\hline
\end{tabular}

time $(P<.001), 43 \%(P<.001)$ of the number of steps, and $38 \%(P<.001)$ of the time in physical activities $\geq 3$ METs. The tubing length was able to explain $22 \%$ $(P=.01)$ of the length of time lying down, and oxygen flow explained $15 \%(P=.040)$ of time spent sitting. On the other hand, percent of predicted $\mathrm{FEV}_{1}$ explained $16 \%$ $(P=.01)$ of walking time variability, $16 \%(P=.033)$ of the number of steps, and 15\% $(P=.04)$ of the time in physical activities $\geq 3$ METs. In the multiple linear regression analysis, only the duration of oxygen therapy (h/d), oxygen flow, and tubing length were maintained in the model as predictors of the level of PADL (Table 4). The correlations between percent of predicted $\mathrm{FEV}_{1}$ and PADL with the time of use of oxygen therapy (h/d) are presented in Figure 2.

\section{Discussion}

In this study, subjects with COPD on home oxygen therapy demonstrated greater limitations and higher levels of dependence in PADL, greater perception of dyspnea, worse health status, and greater number of exacerbations per year than subjects with similar lung function who did not use oxygen therapy. In addition, these subjects spent less time walking and less time on active physical activities and physical activities $\geq 3$ METs, showed lower walking intensity, took fewer steps, and spent more time on physical activities $<1.5$ METs. The use of oxygen therapy was associated with severe physical inactivity, and the duration of oxygen therapy (h/d) was the strongest predictor of PADL. 
Table 3. Comparison of Physical Activities in Daily Life

\begin{tabular}{|c|c|c|c|c|c|c|}
\hline \multirow{2}{*}{$\begin{array}{l}\text { Physical Activities } \\
\text { in Daily Life }\end{array}$} & \multicolumn{3}{|c|}{ Domiciliary Oxygen Therapy Group } & \multicolumn{3}{|c|}{ Control Group } \\
\hline & $\begin{array}{c}\text { Total } \\
(n=29)\end{array}$ & $\begin{array}{c}\text { GOLD III } \\
(n=6)\end{array}$ & $\begin{array}{l}\text { GOLD IV } \\
(n=23)\end{array}$ & $\begin{array}{c}\text { Total } \\
(n=30)\end{array}$ & $\begin{array}{c}\text { GOLD III } \\
(n=6)\end{array}$ & $\begin{array}{c}\text { GOLD IV } \\
(n=24)\end{array}$ \\
\hline Sitting time, min & $418 \pm 104$ & $419 \pm 65.6$ & $418 \pm 114$ & $390 \pm 111$ & $358 \pm 79.4$ & $398 \pm 117$ \\
\hline Lying time, min & $114 \pm 81.6$ & $104 \pm 108$ & $117 \pm 75.7$ & $103 \pm 85.4$ & $115 \pm 114$ & $101 \pm 79.3$ \\
\hline Standing time, min & $139 \pm 64.3$ & $137 \pm 62.3$ & $140 \pm 66.3$ & $149 \pm 60.9$ & $141 \pm 50.0$ & $151 \pm 64.1$ \\
\hline Walking time, $\min$ & $36.1 \pm 23.7 *$ & $49.3 \pm 31.3 \dagger$ & $33.2 \pm 20.8 \ddagger$ & $90.7 \pm 39.6$ & $131 \pm 32.9 \S$ & $80.6 \pm 34.8$ \\
\hline Time in APA, min & $187 \pm 77.9^{*}$ & $197 \pm 74.0$ & $184 \pm 80.4 \neq$ & $258 \pm 91.4$ & $291 \pm 87.5$ & $249 \pm 92.4$ \\
\hline Time in SPA, min & $533 \pm 77.8$ & $523 \pm 74,0$ & $535 \pm 80.3$ & $494 \pm 83.1$ & $473 \pm 77.0$ & $499 \pm 85.3$ \\
\hline $\begin{array}{l}\text { Intensity of movement of the } \\
\text { walk, } \mathrm{m} / \mathrm{s}^{2}\end{array}$ & $1.53 \pm 0.22 *$ & $1.54 \pm 0.30$ & $1.53 \pm 0.21$ & $1.88 \pm 1.01$ & $2.00 \pm 0.44$ & $1.85 \pm 1.11$ \\
\hline Energy expenditure, METs & $1,098 \pm 225$ & $1,166 \pm 146$ & $1,080 \pm 242$ & $1,184 \pm 225$ & $1,401 \pm 247 \S$ & $1,129 \pm 188$ \\
\hline Steps, no./d & $2,669 \pm 1,883^{*}$ & $3,547 \pm 22,617 \dagger$ & $2,429 \pm 1,628 \ddagger$ & $4,599 \pm 2,308$ & $7,190 \pm 1,952 \S$ & $3,951 \pm 1,922$ \\
\hline $\begin{array}{l}\text { Time in physical activities } \\
\geq 3 \text { METs }\end{array}$ & $47.8 \pm 29.6^{*}$ & $63.8 \pm 40.4$ & $43.4 \pm 24.9 \ddagger$ & $75.0 \pm 28.5$ & $103 \pm 28.2 \S$ & $67.9 \pm 24.4$ \\
\hline $\begin{array}{l}\text { Time in SPA }<1.5 \text { METs, } \\
\text { min }\end{array}$ & $623 \pm 63.7 *$ & $643 \pm 43.5$ & $617 \pm 68.0$ & $598 \pm 50.0$ & $577 \pm 70.6$ & $603 \pm 44.0$ \\
\hline $\begin{array}{l}\text { Results are presented as mean } \pm \mathrm{SD} \text {. } \\
* P<.05 \text { vs. total control group. } \\
\dagger P<.05 \text { vs. GOLD III controls. } \\
¥ P<.05 \text { vs. GOLD IV controls. } \\
\S P<.05 \text { vs. GOLD IV controls. } \\
\text { APA }=\text { active physical activities } \\
\text { SPA }=\text { sedentary physical activities } \\
\text { MET = metabolic equivalent of task }\end{array}$ & & & & & & \\
\hline
\end{tabular}

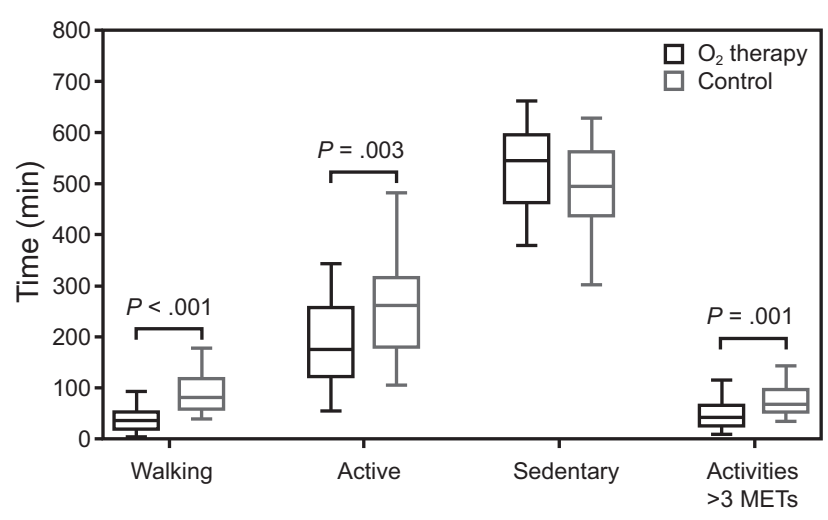

Fig. 1. Box-plot for comparison between domiciliary oxygen therapy group and controls in physical activities in daily life. METs = metaboloic equivalent of tasks.

Previous studies have reported that individuals with COPD are less active in daily life than healthy elderly individuals, ${ }^{29}$ that there is a difference in the level of PADL among the GOLD spirometric classifications, ${ }^{12,34}$ and that the low level of physical activity is associated with the use of oxygen therapy. ${ }^{8,9}$ It is well known that individuals with COPD who undergo oxygen therapy tend to have lower $\mathrm{FEV}_{1} \cdot{ }^{35}$ However, no studies have so far compared subjects using or not using oxygen therapy through careful attention to reduce the influence of pulmonary function on the results. At this time, it has not been established whether the reduction in the level of PADL is influenced by the use of oxygen therapy or the worse pulmonary function of these individuals.

The results of this study indicate that subjects with COPD using home oxygen therapy take fewer steps and walk less and more slowly in daily life than subjects who do not use oxygen therapy. In the control group, GOLD IV subjects with COPD showed less walking time, less energy expenditure, fewer number of steps, and less time in physical activities $\geq 3$ METs when compared to GOLD III subjects with COPD $(P<.05$, Table 3$)$; similar results not observed in the home oxygen therapy group. This suggests that the reduction in PADL in subjects who use home oxygen therapy may occur regardless of the severity of the disease. Although percent of predicted $\mathrm{FEV}_{1}$ is considered a predictor of PADL level, we observed that the time of oxygen therapy per day was a better predictor of walking time, number of steps, and time in physical activities $\geq 3$ METs. In addition, we observed that the longer the duration of oxygen therapy use, the less time subjects spent walking and engaging in physical activities $\geq 3$ METs and the lower the number of steps taken, findings which indicate that the reduction of the level of physical activity goes along with the severity of the disease and the need for greater oxygen supplementation. The development of strategies to slow the progression of the disease and to prevent further exacerbations is very important in this 
Table 4. Multiple Regression Models for the Level of Physical Activity

\begin{tabular}{|c|c|c|c|c|}
\hline & Regression Coefficient & Standard Error $(95 \% \mathrm{CI})$ & $\mathrm{R}^{2}$ & $P$ \\
\hline \multicolumn{5}{|l|}{ Walking time } \\
\hline Constant & 89.7 & $13.0(63.0$ to 116$)$ & NA & $<.001$ \\
\hline Oxygen therapy time (h/d) & -2.92 & $0.68(-4.33$ to -1.52$)$ & 0.41 & $<.001$ \\
\hline \multicolumn{5}{|l|}{ Steps, no./d } \\
\hline Constant & 7,035 & $1,016(4,946$ to 9,123$)$ & NA & $<.001$ \\
\hline Oxygen therapy time (h/d) & -238 & $53.4(-348$ to -128$)$ & 0.43 & $<.001$ \\
\hline \multicolumn{5}{|c|}{ Time in physical activities $\geq 3$ METs } \\
\hline Constant & 111 & 16.4 (77.7 to 145$)$ & NA & $<.001$ \\
\hline Oxygen therapy time (h/d) & -3.48 & $0.86(-5.25$ to -1.70$)$ & 0.38 & $<.001$ \\
\hline \multicolumn{5}{|l|}{ Sitting time } \\
\hline Constant & 376 & $75.3(220$ to 531$)$ & NA & $<.001$ \\
\hline Oxygen flow & 57.4 & 25.4 (5.02 to 110$)$ & 0.28 & .033 \\
\hline Tubing length, $\mathrm{m}$ & -11.2 & $5.19(-21.9$ to -0.56$)$ & 0.28 & .040 \\
\hline \multicolumn{5}{|l|}{ Lying time } \\
\hline Constant & 23.6 & $36.4(-51.2$ to 98.5$)$ & NA & .52 \\
\hline Tubing length, $\mathrm{m}$ & 11.2 & 4.15 (2.64 to 19.7$)$ & 0.22 & .01 \\
\hline
\end{tabular}

$\mathrm{NA}=$ not applicable

MET $=$ metabolic equivalent of task
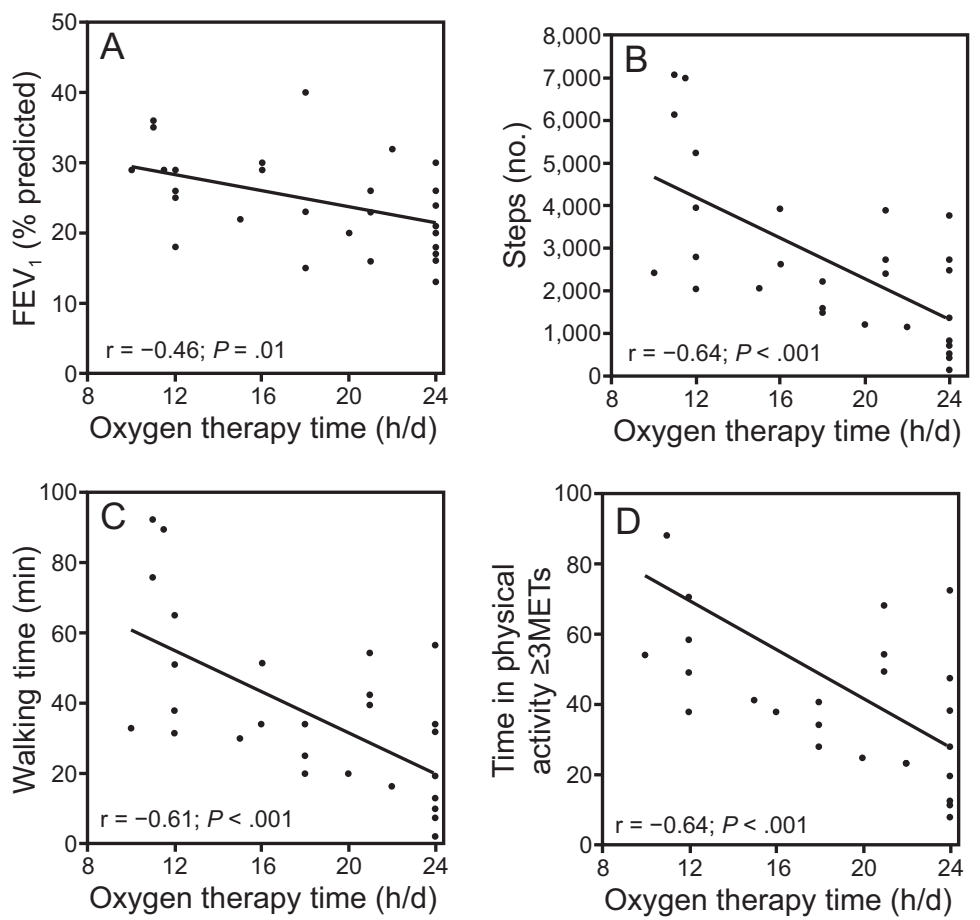

Fig. 2. Correlations between (A) FEV1\% predicted, (B) number of steps, (C) walking time, and (D) time in physical activity $\geq 3$ METS with time of use of oxygen therapy $(\mathrm{h} / \mathrm{d})$. METs $=$ metabolic equivalent of tasks.

population, especially for patients who spend a reduced number of hours per day on oxygen therapy to avoid the progression of the reduction of the level of physical activity.

Oxygen flow during oxygen supplementation and tubing length were predictors of time spent sitting, and tubing length was a predictor of time spent lying down. The lower functional performance of these subjects is perhaps more likely to be justified by the restriction imposed by the device used in oxygen supplementation than by the greater impairment of lung function. Previous studies have already hypothesized that the additional burden imposed by 


\section{Home Oxygen and Physical Activity in COPD}

the equipment and the type of device used for oxygen supplementation, especially in more functionally compromised individuals, may negatively influence the practice and performance of PADL. ${ }^{36}$

In addition to this hypothesis, it is reported that inadequate guidance to these patients on oxygen tubing length may negatively influence their PADL and quality of life. ${ }^{37}$ Many companies eventually restrict tubing length at short distances because they mistakenly believe that this can affect the flow of oxygen which contributes to the reduction of the mobility of these patients. Aguiar et al ${ }^{37}$ verified that a length of $30 \mathrm{~m}$ does not alter the flow and fractions of inspired oxygen supplied, and it may provide greater freedom for patients to move around at home. In this study, we observed that the mean tubing length was $9.5 \mathrm{~m}$, which may had a direct influence on the low level of PADL in subjects in the home oxygen therapy group.

Another important finding was that both groups had a high number of subjects with sedentary behaviors. Sedentary behavior differs from physical inactivity because it is related to activities with an intensity of $<1.5$ METs in sitting or reclining positions. ${ }^{32,38,39}$ Thus, individuals may reach high levels of physical activity, but they spend a great part of the day performing low-energy expenditure physical activities (ie, physical activities $<1.5$ METs), thus presenting sedentary behavior. ${ }^{32}$ In recent years, attention has been paid to the adverse effects of sedentary lifestyle. ${ }^{40,41}$ Recent studies have demonstrated that sedentary behavior is related to mortality in the general population ${ }^{42}$ and in subjects with COPD. ${ }^{33}$ In this study, we observed that subjects in the home oxygen therapy group spent less time on active physical activities and physical activities $\geq 3$ METs and had a higher frequency of severe physical inactivity, whereas there was no difference between the groups regarding the time spent in sedentary activities and the frequency of sedentary behavior when compared to those using oxygen therapy. Therefore, it may be concluded that subjects with COPD have sedentary behavior regardless of the use of oxygen therapy. However, those who use oxygen therapy have lower levels of PADL. Notably, the level of physical activity is another strong predictor of mortality in COPD patients, ${ }^{43}$ and the fact that patients who use oxygen therapy are less active in daily life suggests an increased risk of death. Thus, it can be inferred that both sedentary lifestyle and physical inactivity may impact the decrease in survival of these patients even more.

In addition to presenting lower levels of physical activity, subjects in the home oxygen therapy group had worse dyspnea on exertion, more functional limitation, greater levels of dependence for PADL, and worse general health status than subjects who did not use oxygen therapy, despite similar pulmonary function between them. These results may suggest a strong impact of chronic hypoxemia on the functional performance of these subjects. ${ }^{12}$ However, no differences were observed between the groups in relation to quadriceps muscle strength, and this was not a determining factor for the level of PADL. Quadriceps muscle resistance may be a factor that is specific to these individuals. Quadriceps muscle resistance was not evaluated in our sample, and it is closely related to time spent walking, active physical activities, physical activities $\geq 3$ METs, and the number of steps. These activities showed differences between the groups. Furthermore, the number of exacerbations per year was higher in the home oxygen therapy group. Notably, COPD exacerbations negatively influence the prognosis of patients $^{16}$ and reduce the level of PADL in COPD patients. ${ }^{44}$ However, in our sample, this was not a determinant factor of the time spent on the evaluated PADL or of the duration of oxygen therapy.

One limitation of the study is the small number of subjects evaluated in the GOLD III stage, which may have influenced the comparisons between GOLD III and GOLD IV. However, this was not the purpose of our study, and matching the groups ensured that classification distributions were similar between them. Another possible limitation is the inability to ensure that the oxygen therapy followed all of the criteria and recommendations for its indication and prescription despite the use of a standardized protocol for indication and follow-up on these patients as presented by the Health Department of the State of Santa Catarina.

This is the first study to assess objectively the level of PADL in subjects with COPD using home oxygen therapy by means of a triaxial accelerometer and compare them to subjects who do not use oxygen therapy and have similar lung function. The findings add relevant knowledge about these subjects' PADL and demonstrate that strategies that facilitate physical activity and modify sedentary behavior should be investigated in these subjects because oxygen therapy alone does not improve levels of PADL. Thus, increasing the physical activity of patients with COPD using oxygen at home seems to be fundamental to promoting an increase in survival and improvement in quality of life. Oxygen supplementation may lead to a restriction to home environment, which may reflect the low demand of these individuals for pulmonary rehabilitation programs or the practice of regular physical activity.

\section{Conclusion}

Subjects with COPD in GOLD stage III and stage IV on home oxygen therapy had lower levels of PADL, greater limitations and higher levels of dependence in PADL, greater perception of dyspnea, and worse health status compared to subjects with COPD and similar pulmonary function who did not use oxygen therapy. The use of home 


\section{Home Oxygen and Physical Activity in COPD}

oxygen therapy is associated with the level of PADL. Pulmonary function influences the level of PADL; however, the time of oxygen therapy per day is a stronger predictor in these subjects. Considering that the level of physical activity is a strong predictor of mortality for patients with COPD, the findings of this study suggest that it is necessary to consider the oxygen therapy factor when investigating PADL in this population.

\section{REFERENCES}

1. Singh SJ, ZuWallack RL, Garvey C, Spruit MA, American Thoracic Society/European Respiratory Society Task Force on Pulmonary R. Learn from the past and create the future: the 2013 ATS/ERS statement on pulmonary rehabilitation. Eur Respir J 2013;42(5):1169-1174.

2. Rabe KF, Watz H. Chronic obstructive pulmonary disease. Lancet 2017;389:1931-1940.

3. Kon SS, Canavan JL, Jones SE, Nolan CM, Clark AL, Dickson MJ, et al. Minimum clinically important difference for the COPD assessment test: a prospective analysis. Lancet Respir Med 2014;2(3):195203.

4. Nocturnal Oxygen Therapy Trial Group. Continuous or nocturnal oxygen therapy in hypoxemic chronic obstructive lung disease: a clinical trial. Ann Intern Med 1980(93):391-398.

5. Ergan B, Nava S. Long-term oxygen therapy in COPD patients who do not meet the actual recommendations. COPD 2017;14(3):351-366.

6. Magnet FS, Storre JH, Windisch W. Home oxygen therapy: evidence versus reality. Expert Rev Respir M 2017;11(6):425-441.

7. Goldstein R, Hill K, Brooks D, Dolmage T. Pulmonary rehabilitation: a review of the recent literature. Chest 2012;142(3):738-749.

8. Garcia-Aymerich J, Felez MA, Escarrabill J, Marrades RM, Morera $\mathrm{J}$, Elosua R, et al. Physical activity and its determinants in severe chronic obstructive pulmonary disease. Med Sci Sports Exerc 2004; 36(10): 1667-1673.

9. Sandland CJ, Singh SJ, Curcio A, Jones PM, Morgan MD. A profile of daily activity in chronic obstructive pulmonary disease. J Cardiopulm Rehabil 2005;25(3):181-183.

10. Okubadejo A, O'Shea L, Jones P, Wedzicha J. Home assessment of activities of daily living in patients with severe chronic obstructive pulmonary disease on long-term oxygen therapy. Eur Respir J 1997; 10(7):1572-1575.

11. Spruit MA, Augustin IM, Vanfleteren LE, Janssen DJ, Gaffron S, Pennings HJ, et al. Differential response to pulmonary rehabilitation in COPD: multidimensional profiling. Eur Respir J 2015;46(6): 16251635.

12. Hartman J, Boezen H, de Greef M, Ten Hacken N. Physical and psychosocial factors associated with physical activity in patients with chronic obstructive pulmonary disease. Arch Phys Med Rehabil 2013; 94(12):2396-2402.

13. Watz H, Pitta F, Rochester CL, Garcia-Aymerich J, ZuWallack R, Troosters T, et al. An official European Respiratory Society statement on physical activity in COPD. Eur Respir J 2014;44(6):15211537.

14. Van Remoortel H, Giavedoni S, Raste Y, Burtin C, Louvaris Z, Gimeno-Santos E, et al. Validity of activity monitors in health and chronic disease: a systematic review. Int J Behav Nutr Phys Act 2012;9:84.

15. Bossenbroek L, de Greef MH, Wempe JB, Krijnen WP, Ten Hacken NH. Daily physical activity in patients with chronic obstructive pulmonary disease: a systematic review. COPD 2011;8(4):306-319.

16. Global Initiative for Chronic Obstructive Lung Disease. Global strategy for the diagnosis, management, and prevention of chronic obstructive pulmonary disease. Available at: https://goldcopd.org/gold-reports/. Accessed August 2, 2016.

17. Hardinge M, Annandale J, Bourne S, Cooper B, Evans A, Freeman $\mathrm{D}$, et al. British Thoracic Society guidelines for home oxygen use in adults. Thorax 2015;70(Suppl 1):i1-43.

18. Hulley SB, Cummings SR, Browner WS, Grady D, Hearst N, Newman TB. Delineando a Pesquisa Clínica, 2nd edition. Porto Alegre: Artmed; 2006.

19. Miller MR, Hankinson J, Brusasco V, Burgos F, Casaburi R, Coates A, et al. Standardisation of spirometry. Eur Respir J 2005;26(2):319-338.

20. Pereira CAC, Sato T, Rodrigues SC. Novos valores de referência para espirometria forçada em brasileiros adultos de raça branca. J Bras Pneumol 2007;33(4):397-406.

21. Kovelis D, Segretti NO, Probst VS, Lareau SC, Brunetto AF, Pitta F. Validation of the Modified Pulmonary Functional Status and Dyspnea Questionnaire and the Medical Research Council scale for use in Brazilian patients with chronic obstructive pulmonary disease. J Bras Pneumol 2008;34(12):1008-1018.

22. Hajiro T, Nishimura K, Tsukino M, Ikeda A, Oga T, Izumi T. A comparison of the level of dyspnea vs disease severity in indicating the health-related quality of life of patients with COPD. Chest 1999; 116(6): 1632-1637.

23. Gupta N, Pinto LM, Morogan A, Bourbeau J. The COPD assessment test: a systematic review. Eur Respir J 2014;44(4):873-884.

24. Andrews AW, Thomas MW, Bohannon RW. Normative values for isometric muscle force measurements obtained with hand-held dynamometers. Phys Ther 1996;76(3):248-259.

25. Katz S, Akpom C. A measure of primary sociobiological functions. Int J Health Serv 1976;6(3):493-508.

26. Vestbo J, Vogelmeier C, Small M, Higgins V. Understanding the GOLD 2011 Strategy as applied to a real-world COPD population. Respir Med 2014;108(5):729-736.

27. Pitta F, Troosters T, Probst VS, Spruit MA, Decramer M, Gosselink R. Quantifying physical activity in daily life with questionnaires and motion sensors in COPD. Eur Respir J 2006;27(5):1040-1055.

28. Hernandez C, Aibar J, de Batlle J, Gomez-Cabrero D, Soler N, Duran-Tauleria E, et al. Assessment of health status and program performance in patients on long-term oxygen therapy. Respir Med 2015;109(4):500-509.

29. Pitta F, Troosters T, Spruit M, Probst V, Decramer M, Gosselink R. Characteristics of physical activities in daily life in chronic obstructive pulmonary disease. Am J Respir Crit Care Med 2005;171(9):972-977.

30. Rabinovich RA, Louvaris Z, Raste Y, Langer D, Van Remoortel H, Giavedoni S, et al. Validity of physical activity monitors during daily life in patients with COPD. Eur Respir J 2013;42(5):1205-1215.

31. Depew ZS, Novotny PJ, Benzo RP. How many steps are enough to avoid severe physical inactivity in patients with chronic obstructive pulmonary disease? Respirology 2012;17(6):1026-1027.

32. Hill K, Gardiner PA, Cavalheri V, Jenkins SC, Healy GN. Physical activity and sedentary behaviour: applying lessons to chronic obstructive pulmonary disease. Intern Med J 2015;45(5):474-482.

33. Furlanetto KC, Donaria L, Schneider LP, Lopes JR, Ribeiro M, Fernandes $\mathrm{KB}$, et al. Sedentary behavior is an independent predictor of mortality in subjects with COPD. Respir Care 2017;62(5):579-587.

34. Watz H, Waschki B, Meyer T, Magnussen H. Physical activity in patients with COPD. Eur Respir J 2009;33(2):262-272.

35. Hartman JE, Boezen HM, de Greef MH, Ten Hacken NH. Physical and psychosocial factors associated with physical activity in patients with chronic obstructive pulmonary disease. Arch Phys Med Rehabil 2013;94(12):2396-2402 e2397.

36. Crisafulli E, Costi S, De Blasio F, Biscione G, Americi F, Penza S, et al. Effects of a walking aid in COPD patients receiving oxygen therapy. Chest 2007;131(4):1068-1074. 


\section{Home Oxygen and Physical Activity in COPD}

37. Aguiar C, Davidson J, Carvalho AK, Iamonti VC, Cortopassi F, Nascimento OA, et al. Tubing length for long-term oxygen therapy. Respir Care 2015;60(2):179-182.

38. Gibbs BB, Hergenroeder AL, Katzmarzyk PT, Lee IM, Jakicic JM. Definition, measurement, and health risks associated with sedentary behavior. Med Sci Sports Exerc 2015;47(6):1295-1300.

39. Cavalheri V, Straker L, Gucciardi DF, Gardiner PA, Hill K. Changing physical activity and sedentary behaviour in people with COPD. Respirology 2016;21(3):419-426.

40. Owen N, Healy GN, Matthews CE, Dunstan DW. Too much sitting: the population health science of sedentary behavior. Exerc Sport Sci Rev 2010;38(3):105-113.
41. Pate RR, O'Neill JR, Lobelo F. The evolving definition of "sedentary." Exerc Sport Sci Rev 2008;36(4):173-178.

42. Bauman AE, Chau JY, Ding D, Bennie J. Too much sitting and cardio-metabolic risk: an update of epidemiological evidence. Curr Cardiovasc Risk Rep 2013;(7):293-298.

43. Waschki B, Kirsten A, Holz O, Muller KC, Meyer T, Watz H, et al. Physical activity is the strongest predictor of all-cause mortality in patients with COPD: a prospective cohort study. Chest 2011; 140(2):331-342.

44. Pitta F, Troosters T, Probst VS, Spruit MA, Decramer M, Gosselink R. Physical activity and hospitalization for exacerbation of COPD. Chest 2006;129(3):536-544. 